\title{
Chronic Hypoxia Impairs Murine Hippocampal Development and Depletes the Postnatal Progenitor Pool by Attenuating Mammalian Target of Rapamycin Signaling
}

\author{
LAKSHMI RAMAN, XIANGMEI KONG, JENNIFER A. GILLEY, AND STEVEN G. KERNIE \\ Departments of Pediatrics and Developmental Biology, University of Texas Southwestern Medical Center, Dallas, Texas 75390
}

\begin{abstract}
Chronic hypoxia $(\mathrm{CH})$ is a major risk factor for impaired cognitive function in various disease states, particularly in the context of cyanotic congenital heart disease. Although most brain development occurs prenatally, the dentate gyrus (DG) of the hippocampus harbors progenitor stem cells that contribute to its ongoing development postnatally. It is unclear how exposure to $\mathrm{CH}$ might affect postnatal hippocampal development, so we used a transgenic mouse that expresses enhanced green fluorescent protein (eGFP) within this progenitor population to determine the effect of $\mathrm{CH}$ on the DG. We find that exposure to $10 \%$ oxygen from postnatal d 3 to 28 results in a smaller DG with long-term impairment of hippocampal neurogenesis. Because the mammalian target of rapamycin (mTOR) pathway is a well-known regulator of cell proliferation and growth and is sensitive to hypoxia, we investigated its activation on exposure to $\mathrm{CH}$ and find it to be attenuated specifically in neural progenitor cells. Systemic inhibition of the mTOR pathway using rapamycin also caused impairment of hippocampal neurogenesis that mimics exposure to $\mathrm{CH}$. Our findings demonstrate that $\mathrm{CH}$ results in long-term impairment of hippocampal neurogenesis and is mediated, in part, by attenuation of the mTOR pathway. (Pediatr Res 70: 159-165, 2011)
\end{abstract}

$\mathrm{M}$ any infants with cyanotic heart disease are in a state of chronic hypoxia $(\mathrm{CH})$ for the first 2 to $3 \mathrm{y}$ of life before repair of the cardiac lesion. $\mathrm{CH}$ that begins in these infants at birth occurs during critical time points of hippocampal development (1). Using a rodent model of $\mathrm{CH}$, we have previously shown a significant alteration in the hippocampal neurometabolome (2) and dendritic arborization in the hippocampus (3). Given the significant alterations in the hippocampus in $\mathrm{CH}$ we investigated how dentate gyrus (DG) neurogenesis, which occurs primarily postnatally, might also be affected. The DG in the hippocampus is one of two well-characterized areas where neurogenesis is known to persist throughout life (4). This region has stem/progenitor cells that are the source of the DG granular neurons $(5,6)$.

The neural stem and progenitor cells in the subgranular layer of the DG have been classified using a neural progenitorspecific nestin-GFP expressing transgenic mouse as type 1, 2a, $2 \mathrm{~b}$, and 3 cells $(7,8)$. The early stem/progenitors (type 1 and 2a) express the neuroepithelial marker nestin, whereas the late

Received October 12, 2010; accepted February 16, 2011.

Correspondence: Lakshmi Raman, M.D., Departments of Pediatrics and Developmental Biology, UT Southwestern Medical Center at Dallas, 5323 Harry Hines Blvd, Dallas, TX 75390; e-mail: lakshmi.raman@UTSouthwestern.edu

Supported by the Perot Family Center for Brain and Nerve Injuries at Children's Medical Center Dallas [L.R.] and NIH grant R01 NS048192 [S.G.K.]. progenitors (type $2 \mathrm{~b}$ and 3 ) down-regulate the expression of nestin and begin to express markers of developing neurons such as the microtubule protein doublecortin (DCX) and polysialated neural cell adhesion molecule (PSA-NCAM) (8). These cells are stimulated in response to environmental enrichment and various other physiological stimuli (9). There is also evidence to suggest that subsets of these progenitor cells are vulnerable to external insults such as hypoxia-ischemia and trauma $(10,11)$.

We wanted to study the effects of $\mathrm{CH}$ on the progenitor stem cell population in the hippocampus and the underlying mechanism involved. To investigate the underlying mechanism of the effects of $\mathrm{CH}$ on neurogenesis, we examined the role of the mammalian target of rapamycin (mTOR) pathway, because hypoxia is an important regulator of the mTOR pathway. Under acute hypoxic conditions, cells regulate the limited energy available by inhibiting energy-intensive processes such as protein translation (12). The inhibition of mTOR, a protein that is required for proper cell proliferation and growth, is the major mechanism through which this is achieved (13). The mTOR pathway plays critical roles in proper brain development including axonal guidance, dendritic development, and dendritic morphogenesis $(14,15)$. mTOR has also been implicated in neurodegenerative disorders such as Alzheimer's Huntington's, and Parkinson's diseases (16-18). We hypothesized that $\mathrm{CH}$ would decrease the progenitor stem pool in the DG of the hippocampus and that this may be mediated through impairment of mTOR signaling.

\section{METHODS}

\begin{abstract}
Animal preparation. The study was approved and conducted according to the guidelines of the Institutional Animal Care and Use Committee at the University of Texas Southwestern Medical Center. The animals were housed and cared for in the Animal Resource Center (ARC) at UT Southwestern Medical Center in compliance with standards for humane and compassionate use of animals in biomedical research. Transgenic mice expressing GFP under the control of the nestin promoter and second intron (nestin-GFP) that were previously generated in our laboratory were backcrossed into the CD1 background for eight generations (19). Offspring were genotyped using the previously published protocol. Nestin-GFP transgenic CD1 mice were used for all experiments. Animals are housed with a 12:12 h dark and light cycle with free access to food and water.
\end{abstract}

Abbreviations: BrdU, 5-Bromo-2-deoxyuridine; CH, chronic hypoxia; DCX, doublecortin; DG, dentate gyrus; 4E-BP1, eukaryotic translation initiation factor; GFP, green fluorescent protein; mTOR, mammalian target of rapamycin 
Induction of $\boldsymbol{C H}$. Dams and their pups were randomly assigned to control or $\mathrm{CH}$ groups. The dams and their pups in the $\mathrm{CH}$ group were housed in a Plexiglas chamber (oxygen concentration $10 \pm 0.1 \%$ ) from postnatal day $(\mathrm{P})$ 3 until d 7, 14, and 28. For P56 experiments, animals in the $\mathrm{CH}$ group were placed in the chamber from P3 to P28 and then in normoxia until d 56. Details outlined in Ref. 2. The pups in the $\mathrm{CH}$ had less body weight by about $40 \%$ at P7, 14, and 28. We have previously demonstrated that the brain weight in this model is reduced in the $\mathrm{CH}$ group (2).

5-Bromo-2-deoxyuridine injection. To determine the proliferation profile of the progenitor stem cell population, a single dose of bromo-2-deoxyuridine (BrdU; $50 \mathrm{mg} / \mathrm{kg}$; Sigma Chemical Co.-Aldrich, St. Louis, MO) dissolved in sterile water was given i.p. 2 h before killing on P7, P14, P28, and P56. This dose captures most of the proliferating cells particularly because these are young animals. To capture the long-term fate of the progenitor stem pool, a separate set of animals in the control and $\mathrm{CH}$ group received a single BrdU injection $(50 \mathrm{mg} / \mathrm{kg})$ given i.p. on P28 and were killed on P56.

Rapamycin injection. To examine the role of pharmacological inhibition of the mTOR pathway on neurogenesis, a separate cohort of animals were given daily injections of rapamycin (LC laboratories, Boston, MA) starting from P5 until P9 at a dose of $50 \mu \mathrm{g} / \mathrm{kg}$ and from P10 to P14 at a dose of 100 $\mu \mathrm{g} / \mathrm{kg}$ i.p. Animals were given $\mathrm{BrdU}(50 \mathrm{mg} / \mathrm{kg})$ i.p. $2 \mathrm{~h}$ before killing.

Immunohistochemistry. Immunohistochemistry (IHC) was performed as outlined in Ref. 20. A DAB protocol was used for stereology measurements and double-labeling immunofluorescence was used to obtain images that are shown. To measure volume, sections obtained from the entire hippocampus were stained with Nissl staining, and volume was measured using MetaView analysis at P14 and P56.

Cell quantification. Unbiased estimates for volume and cell counts were obtained using stereological quantification on an Olympus BX51 System Microscope with a MicroFIRE A/R camera (Optronics). An unbiased counting frame was used with the help of the Optical Fractionator Probe within the Stereo Investigator software. For GFP and DCX cell counting, a $100 \times, 1.4$ NA oil immersion lens was used, and for BrdU cell counting, a 40×, 0.75 NA lens was used. A minimum of 350 cells per animal was counted. The average number of sections was 8 to 10 per animal, and average thickness of the section after processing was approximately $30 \mu \mathrm{m}$. The optical dissector height was $24 \mu \mathrm{m}$ with $3 \mu \mathrm{m}$ guard zone on either side. For counting frame size refer to Table 1 .

To reduce bias between samples, a number of precautions were taken. All samples were processed in a similar manner. To provide room for shrinkage of tissues after processing, average mounting thickness of $30 \mu \mathrm{m}$ was used. Every sixth section was used for counting, and only animals where all the sections were present were used for quantification. The average Schaffer coefficient of error for each staining and time point is mentioned in Table 1. Images shown were obtained by Zeiss LSM 510 confocal microscope using Argon 488 and He 633 lasers.

Western blot. Cortex was dissected out in both the control and $\mathrm{CH}$ groups at P14, homogenized. Twenty micrograms of protein was loaded on $12 \%$ SDS-PAGE for p70 ribosomal S6 protein kinase (S-6) and total S-6 kinase blot and 10\% SDS-PAGE for total and phosphorylated elf-4E-binding protein (4E-BP1) blot. Antibodies used included rabbit anti-phospho-S-6, total S-6 (cell signaling) at 1:1000, rabbit anti-phospho 4E-BP1 and total 4E-BP1(cell signaling) at 1:1000 and rabbit anti $\beta$-tubulin (sigma) at 1:30,000. All secondary antibodies were raised in goat serum (Santa Cruz) and used in dilution of $1: 10,000$.

Protein concentrations were measured (BCA protein assay kit), gels were run and protein transferred to a polyvinylidene fluoride (PVDF) membrane.
After membranes were blocked with 4\% BSA for $1 \mathrm{~h}$ they were incubated with primary antibody overnight at $4^{\circ} \mathrm{C}$ followed by secondary antibody for $2 \mathrm{~h}$ at room temperature. Membranes were washed with PBS-1\% Tween. Protein bands were visualized and quantified by densitometry analysis using Kodak Molecular Imaging Software 4.0.3 and the quantity adjusted to the total protein and anti- $\beta$-tubulin density.

Real-time PCR. DG was microdissected from the hippocampus on P14 in both control and $\mathrm{CH}$ groups. Cell sorting and RNA extraction was done as outlined in Ref. 20. The primer sequence is as follows: GAPDH forward: 5'-CTC AAC TAC ATG GTC TAC ATG TTC CA-3'; GAPDH reverse 5'CCA TTC TCG GOC TTG ACT GT-3'; S6 forward: 5' -TCG GCT GTG TCA AGA TGA AG-3'; S6 reverse 5' CAT GGA AAA ACC TTG CTT GT-3'. Real-time quantification was analyzed on the Applied Biosystems 7,500 Real-time PCR system software.

Statistics. Unpaired $t$ test with unequal variance was used to compare the effect of $\mathrm{CH}$ on progenitor stem cell population at each time point. Paired $t$ test with equal variance was used to compare the effect of $\mathrm{CH}$ on the mTOR pathway by Western and real-time PCR. The data are presented as mean \pm $\mathrm{SD}$, and statistical significance was set at $p<0.05$.

\section{RESULTS}

CH decreases the progenitor pool. To quantify the progenitor stem cell population within the DG, design-based stereology was used to estimate the number of early progenitors (GFP-expressing cells) and actively dividing (BrdUexpressing) cells in nestin-GFP transgenic mice at P7, P14, and P28. Late progenitors of the DG were estimated similarly at P14 by quantifying DCX-expressing cells.

After $4 \mathrm{~d}$ of hypoxic exposure (10\% oxygen from P3-P7), mice were given one dose of BrdU to see how overall proliferation was affected. We observed that BrdU-expressing cells within the DG were reduced by $\sim 50 \%(14,240 \pm 3,276)$ when compared with normoxic controls $(24,109 \pm 3,871$; Fig. $1 C$, $D$, and $T$ ). To then determine whether these dividing cells were stem/progenitors that express GFP, we performed stereological counts and found that progenitors within the dentate were reduced similarly by almost $40 \%$ in the $\mathrm{CH}$-exposed group $(34,562 \pm 1,389)$ compared with the control $(48,789 \pm$ 1,424; Fig. $1 A, B$, and $S$ ).

To determine how $\mathrm{CH}$-induced changes in proliferation progressed over time, we studied animals at P14 and at P28. Here, proliferation as evidenced by BrdU incorporation was also decreased, although not as dramatically as what was observed at P7. Overall proliferation was decreased by $\sim 15 \%$ at P14 (8554 \pm 667 in control versus $7361 \pm 783$ in $\mathrm{CH}$; Fig. $1 I, J$, and $T)$ and at P28 (2398 \pm 254 in control versus $2043 \pm$ 138 in $\mathrm{CH}$; Fig. $1 O, P$, and $T$ ). GFP-expressing progenitors

Table 1. Grid size, average number of cells, and coefficient of error for BrdU, GFP, and DCX at different time points

\begin{tabular}{|c|c|c|c|c|c|c|c|}
\hline \multirow[b]{2}{*}{ Age } & \multirow[b]{2}{*}{ Condition } & \multicolumn{2}{|c|}{$\mathrm{BrDU}($ Grid size $100 \times 100)$} & \multicolumn{2}{|c|}{ GFP $($ Grid size $150 \times 200)$} & \multicolumn{2}{|c|}{ DCX $($ Grid size $150 \times 200)$} \\
\hline & & $\begin{array}{l}\text { Average number } \\
\text { of cells per } \\
\text { sampling site }\end{array}$ & $\begin{array}{l}\text { Coefficient } \\
\text { of error } \\
(\%)\end{array}$ & $\begin{array}{l}\text { Average number } \\
\text { of cells per } \\
\text { sampling site }\end{array}$ & $\begin{array}{l}\text { Coefficient } \\
\text { of error } \\
(\%)\end{array}$ & $\begin{array}{l}\text { Average number } \\
\text { of cells per } \\
\text { sampling site }\end{array}$ & $\begin{array}{c}\text { Coefficient } \\
\text { of error } \\
(\%)\end{array}$ \\
\hline \multirow[t]{2}{*}{ P-7 } & Control $n=6$ & 4.5 & 6 & 3.5 & 8 & NA & \\
\hline & $\mathrm{CH} n=6$ & 2.3 & 8 & 3 & 9.5 & & \\
\hline \multirow[t]{2}{*}{ P-14 } & Control $n=7$ & 1.5 & 9.5 & 2.5 & 7.5 & 4.5 & 9.5 \\
\hline & $\mathrm{CH} n=6$ & 1.5 & 9 & 2 & 11.5 & 3.5 & 7.5 \\
\hline \multirow[t]{2}{*}{ P-28 } & Control $n=6$ & 0.5 & 11 & 1.5 & 6 & $\mathrm{NA}$ & \\
\hline & $\mathrm{CH} n=7$ & 0.5 & 9 & 1.5 & 7 & & \\
\hline \multirow[t]{2}{*}{ P-56 (BrDU injected on P28) } & Control $n=6$ & 0.5 & 8 & \multirow{2}{*}{\multicolumn{2}{|c|}{ NA }} & \multirow{2}{*}{\multicolumn{2}{|c|}{ NA }} \\
\hline & $\mathrm{CH} n=5$ & 0.5 & 8 & & & & \\
\hline \multirow[t]{2}{*}{ P-56 (BrDU injected on P56) } & Control $n=5$ & 0.5 & 10 & \multirow{2}{*}{\multicolumn{2}{|c|}{ NA }} & \multirow{2}{*}{\multicolumn{2}{|c|}{ NA }} \\
\hline & $\mathrm{CH} n=5$ & 0.5 & 10.5 & & & & \\
\hline
\end{tabular}



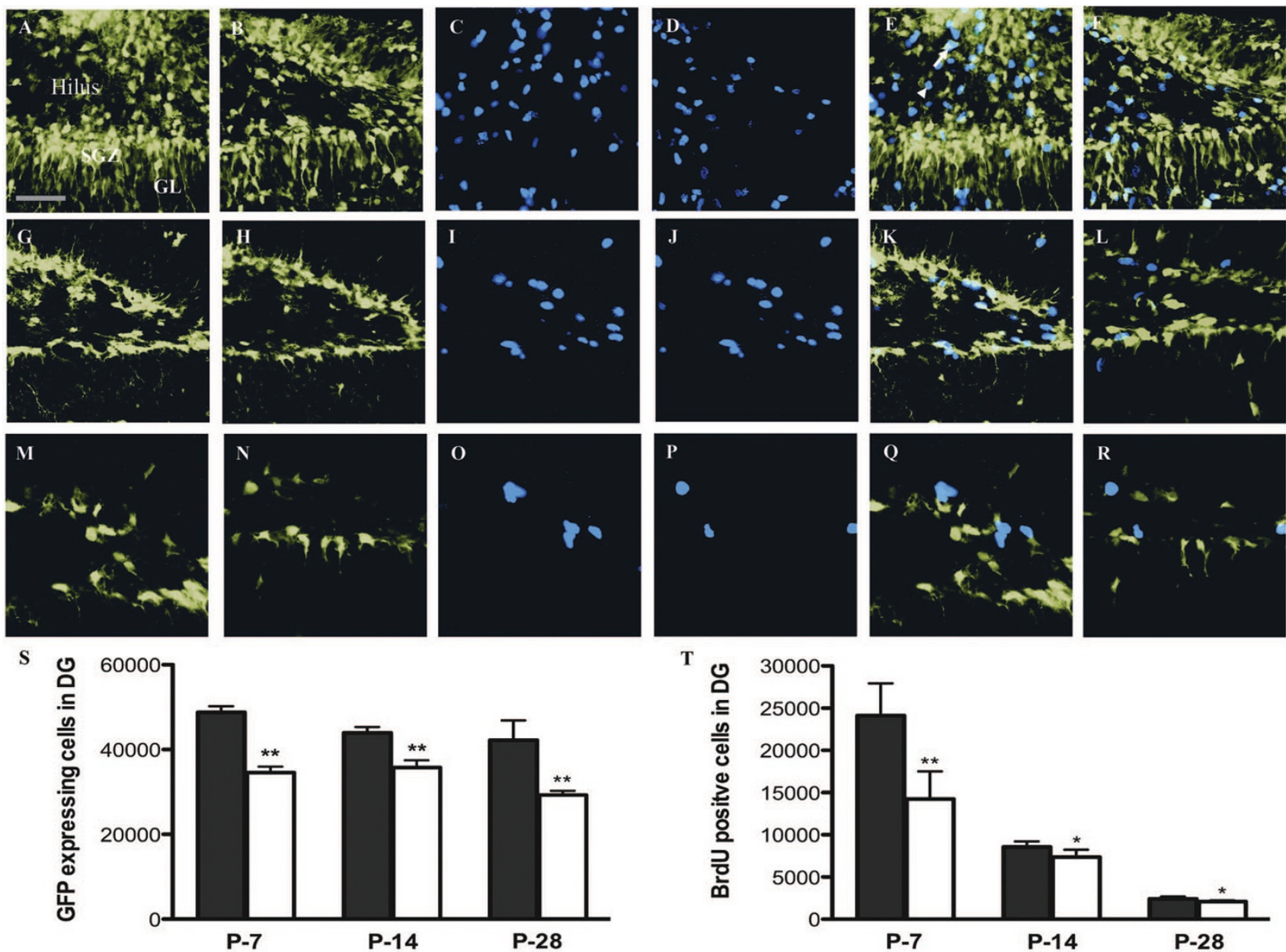

Figure 1. Changes in the progenitor cell population in $\mathrm{CH}$ within the DG from $\mathrm{P} 7$ to P28. Representative pictures of GFP and BrdU expression in DG from control nestin-GFP mice (P7: $A$ and $C ; \mathrm{P} 14: G$ and $I ; \mathrm{P} 28: M$ and $O$ ) and nestin-GFP mice exposed to CH (P7: $B$ and $D ; \mathrm{P} 14: H$ and $J ; \mathrm{P} 28: N$ and $P$ ) respectively, showing significantly decreased numbers of GFP-positive $(S)$ and BrdU-positive cells $(T)$ in the $\mathrm{CH}$-exposed mice as quantified by design-based stereology. The merged image in the control (P7: $E$; P14: $K$; P28: $Q$ ) and CH (P7: F; P14: $L ; \mathrm{P} 28: R$ ) shows BrdU-positive cells that are colocalized with GFP (arrows) and

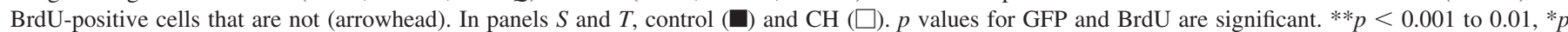
0.01 to 0.05 , respectively. Error bars represent SD, and the scale bar represents $35 \mu \mathrm{M}$. GL, granular layer and SGZ, subgranular zone.

were also decreased by $\sim 20 \%$ at P14 $(43,965 \pm 1,365$ in control versus $35,793 \pm 1,662$ in $\mathrm{CH}$; Fig $1 G, H$, and $S$ ) and $30 \%$ at P $28(42,198 \pm 4,598$ in control versus $29,254 \pm 943$ in $\mathrm{CH}$; Fig $1 M, N$, and $S$ ), which was more pronounced than the decrease seen at P14. This is in marked contrast to the $40 \%$ reduction seen at $\mathrm{P} 7$.

CH impairs DG development. Because $\mathrm{CH}$ impairs overall proliferation and decreases the number of GFP-expressing progenitors at all time points examined (Fig. $1 B, H, N$, and $S$ ), we next wanted to determine whether the DG itself developed aberrantly. To determine this, we measured the volume of the neuronal layer of the DG at P14. We observed that the volume of the DG was significantly decreased by $\sim 20 \%(6 \pm 2.6 \times$ $10^{8} \mu \mathrm{M}$ in control versus $4.7 \pm 0.2 \times 10^{8} \mu \mathrm{M}$ in $\mathrm{CH}$; Fig. $2 A-D, G)$ in the $\mathrm{CH}$ group, which correlates well with the $20 \%$ reduction of proliferation observed at that time point (Fig. $1 I, J$, and $T$ ).

We also wanted to determine whether this overall loss in neuronal volume could be explained by fewer late progenitors as labeled by DCX expression. Using unbiased stereology, we determined that DCX-expressing late progenitors at P14 were decreased by $\sim 22 \%(64,316 \pm 7,801$ in control versus $50,084 \pm 7,854$ in $\mathrm{CH}$; Fig. $2 E, F$, and $H$ ). Again, this correlates well with the overall decrease in the DG volume that is observed at this time point.

Recovery of $D G$ volume occurs after 4 wk of normoxia but proliferation remains impaired. Immediately after exposure to $\mathrm{CH}$, mice demonstrated stunted growth that normalized after exposure to normoxia. To determine whether a similar recovery occurs in the DG, we quantified the number of early progenitors (GFP expressing) at P56 and found that these numbers had normalized after the animals had been in normoxia for $4 \mathrm{wk}(27,720 \pm 2,781$ in control versus $25,752 \pm$ 3,345 in $\mathrm{CH}$ ) Similarly, when the DG volume was measured at P56, the group exposed to hypoxia from P3-P28 normalized its DG volume compared with normoxic controls (5.2 \pm $0.42 \times 10^{8} \mu \mathrm{M}$ in control versus $5.2 \pm 0.12 \times 10^{8} \mu \mathrm{M}$ in $\mathrm{CH})$. Figure 3 demonstrates that immediately after exposure to normoxia at P28, there was a rebound of BrdU-expressing cells within the DG $(1710 \pm 162$ in control versus $2146 \pm 215$ in $\mathrm{CH}$ ) (Fig. $3 A, B$, and $E$ ). However, when mice exposed to hypoxia from P3-P28 were analyzed for proliferation at P56 

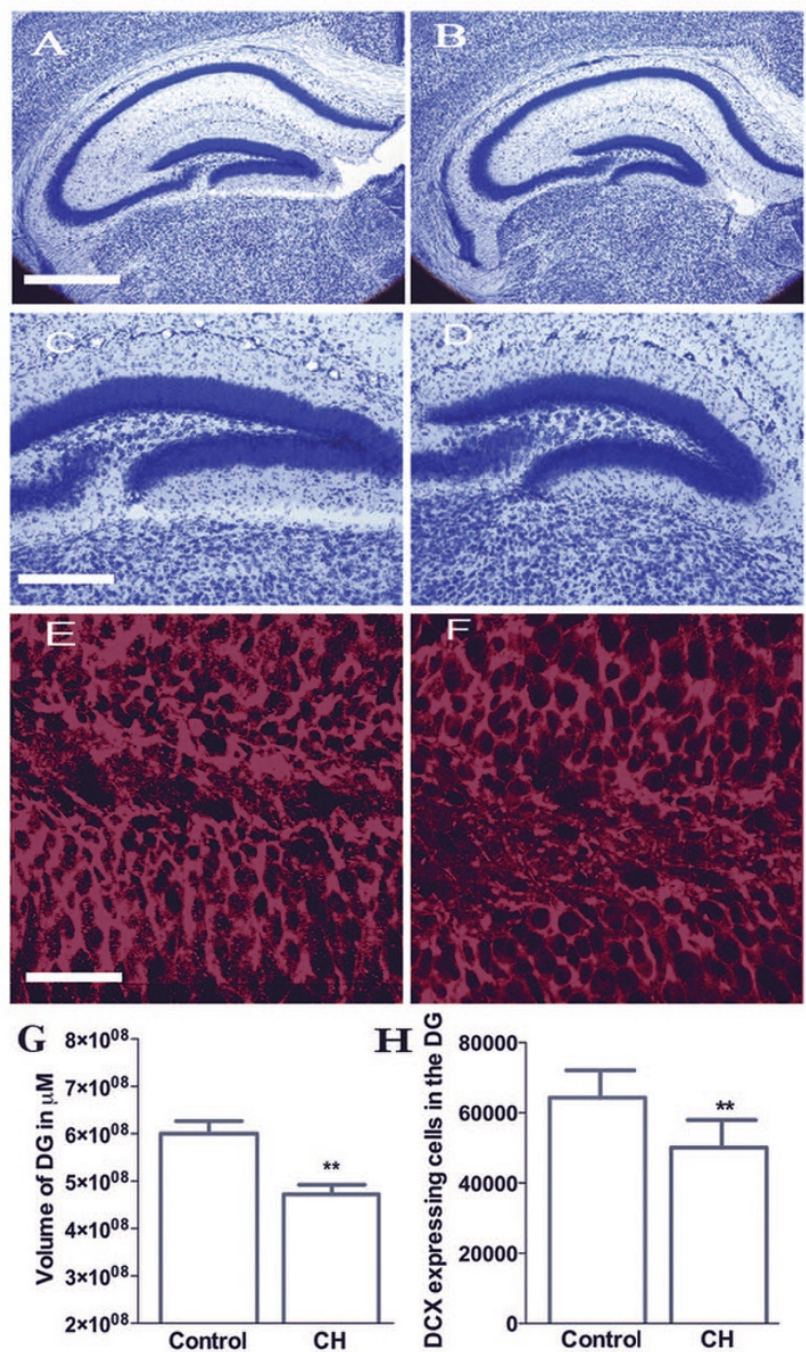

Figure 2. Decreased volume of the DG and DCX-expressing late progenitors at P14 after $\mathrm{CH}$. Representative pictures of Nissl staining in control $(A$ and $C)$ and $\mathrm{CH}(B$ and $D)$ mice at lower $(A$ and $B)$ and higher magnification $(C$ and $D$ ) showing the volume of the DG. Volume estimation by MetaView analysis shows significantly decreased volume in the $\mathrm{CH}$ group $(G)$. ** $p<$ 0.001. Error bars represent SD, and scale bars represent $200 \mu \mathrm{M}(A$ and $B)$ and $100 \mu \mathrm{M}(C$ and $D)$. Representative pictures of DCX staining in the DG at $\mathrm{P} 14$, in the control $(E)$ and $\mathrm{CH}(F)$ showing significantly decreased number of cells under $\mathrm{CH}(H)$ as quantified by design-based stereology. ${ }^{* *} p=0.001$ to 0.01 . Error bars represent SD, and scale bars represent $35 \mu \mathrm{M}$.

by BrdU given just before sacrifice, there was a marked attenuation of proliferating cells in the mature DG $(2103 \pm$ 248 in control versus $1393 \pm 161$ in $\mathrm{CH}$; Fig. $3 C, D$, and $F$ ). This suggests long-term impairment of the proliferative potential in the adult.

The mTOR pathway is impaired in $\mathrm{CH}$. The mechanisms underlying $\mathrm{CH}$-dependent attenuation of progenitors remain unclear but may involve mTOR. Two substrates in the mTOR pathway are S6 and 4E-BP1, which undergo mTOR-mediated phosphorylation and are used as readouts for pathway activation (13). We performed Western blot analysis on isolated cortex from hypoxia-exposed animals and determined that both phospho-S6 and phospho-4E-BP1 levels were attenuated in the cortex (Fig. 4A-F). To determine the cell specificity of this effect, we used fluorescent activated cell-sorting (FACS)
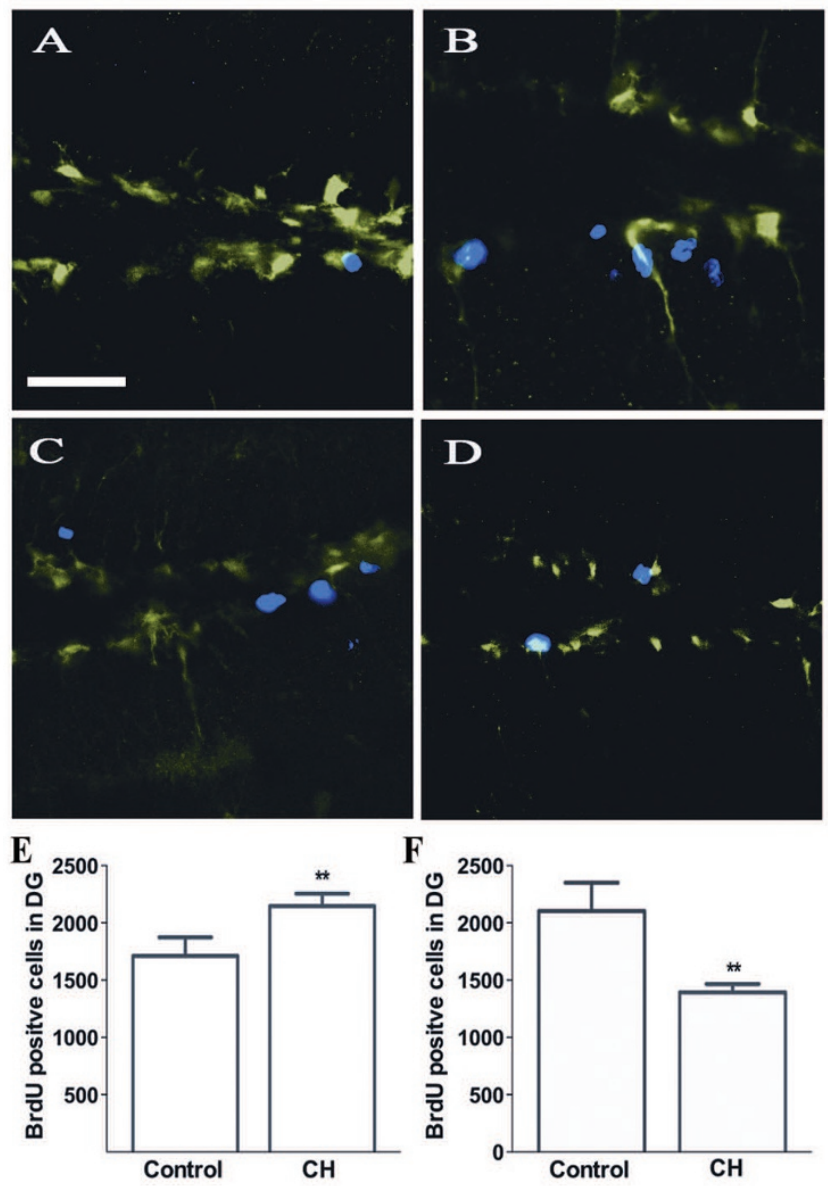

Figure 3. Changes in the number of actively dividing cells within the DG at P56 after $\mathrm{CH}$. Representative pictures of GFP and BrdU expression in the DG of mice under control $(A)$ and $\mathrm{CH}$ conditions $(B)$ shows a significant increase in the number of the BrdU-positive cells in the $\mathrm{CH}$ mice $(E)$ as measured by design-based stereology. Mice were injected with BrdU at P28 just after being removed from hypoxic conditions and quantified 4 wk later. Representative pictures of BrdU staining under control $(C)$ and $\mathrm{CH}$ conditions $(D)$ showing decreased number of BrdU-positive cells under $\mathrm{CH}(F)$ as measured by design-based stereology. Mice were injected with BrdU $2 \mathrm{~h}$ before perfusion at P56. $* * p=0.001$ to 0.01. Error bars represent SD, and scale bars represent $35 \mu \mathrm{M}$.

to isolate GFP-expressing progenitors from the DG of both control and $\mathrm{CH}$-exposed mice. We quantified S6 mRNA levels in GFP-positive cells using quantitative PCR and found that mRNA expression is reduced by $\sim 50 \%$ in the $\mathrm{CH}$ group at both P7 and P14 and persists at P28 (Fig. 4G). These data suggest that the mTOR pathway may be involved in the pathology of the aberrant hippocampal development that accompanies $\mathrm{CH}$ and that its attenuation is specific to the progenitor population.

mTOR inhibition by rapamycin decreases the progenitor stem cell pool. We next investigated whether pharmacologically inhibiting the mTOR pathway using rapamycin phenocopies $\mathrm{CH}$. Nestin-GFP animals were injected with rapamycin from $\mathrm{P} 5$ to P14 and killed $2 \mathrm{~h}$ after an injection of BrdU. Using unbiased stereology, we quantified GFP-expressing early progenitors, DCX-expressing late progenitors, and the actively dividing cells by BrdU incorporation on P14. Animals treated with rapamycin had significantly decreased numbers of both 

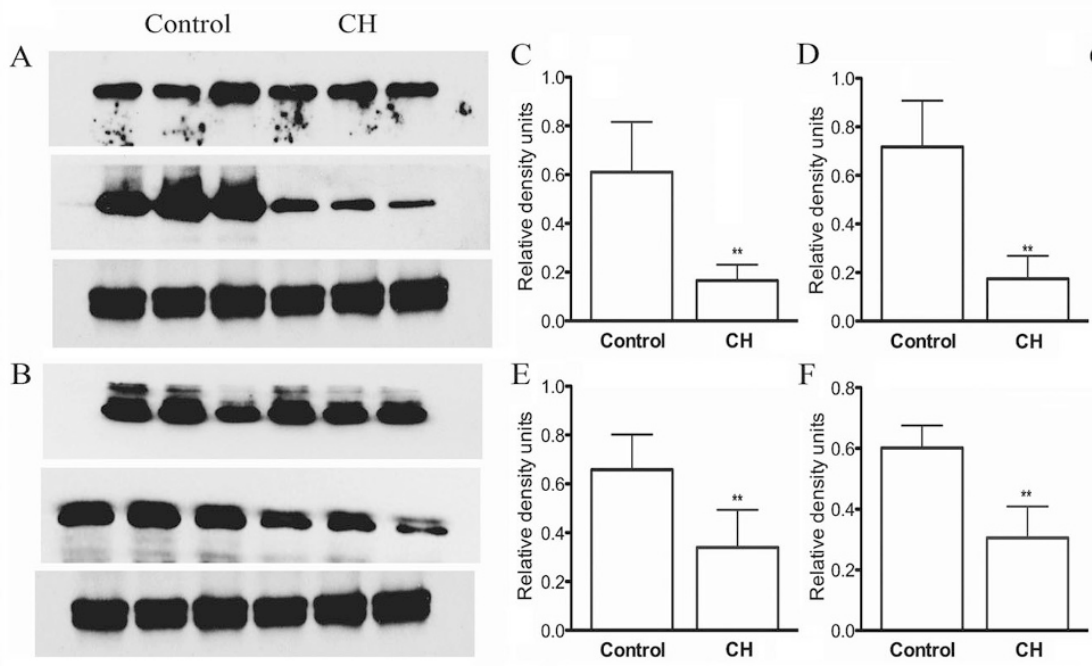

G

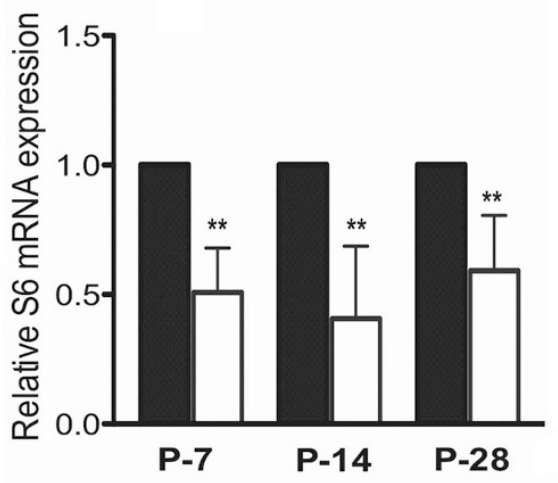

Figure 4. Decreased phosphorylated S6 and 4E-BP1 levels in $\mathrm{CH}$ in the cortex. (A) Western blot of total (top), phosphorylated (p) S6 (middle) and tubulin (bottom) showing decreased concentrations of pS6 under $\mathrm{CH}$ compared with controls. (B) Western blot of total (top), phosphorylated (p) 4E-BP1 (middle) and tubulin (bottom) showing decreased concentrations of p4E-BP1 in $\mathrm{CH}$ compared with the controls. $(C)$ and $(D)$ showing significantly decreased concentrations of pS6 compared with total S6 and tubulin, respectively. $(E)$ and $(F)$ show significantly decreased concentrations of p4E-BP1 compared with total 4E-BP1 and tubulin, respectively. $(G)$ Significantly decreased expression of S6 mRNA expression is observed at all three time points in the $\mathrm{CH}$ group ( $\square$ ) from the FACS-sorted GFP-expressing DG progenitor cells $(\square) . * * p=0.001$ to 0.01 and $* p=0.01$ to 0.05 , respectively. Error bars represent SD.
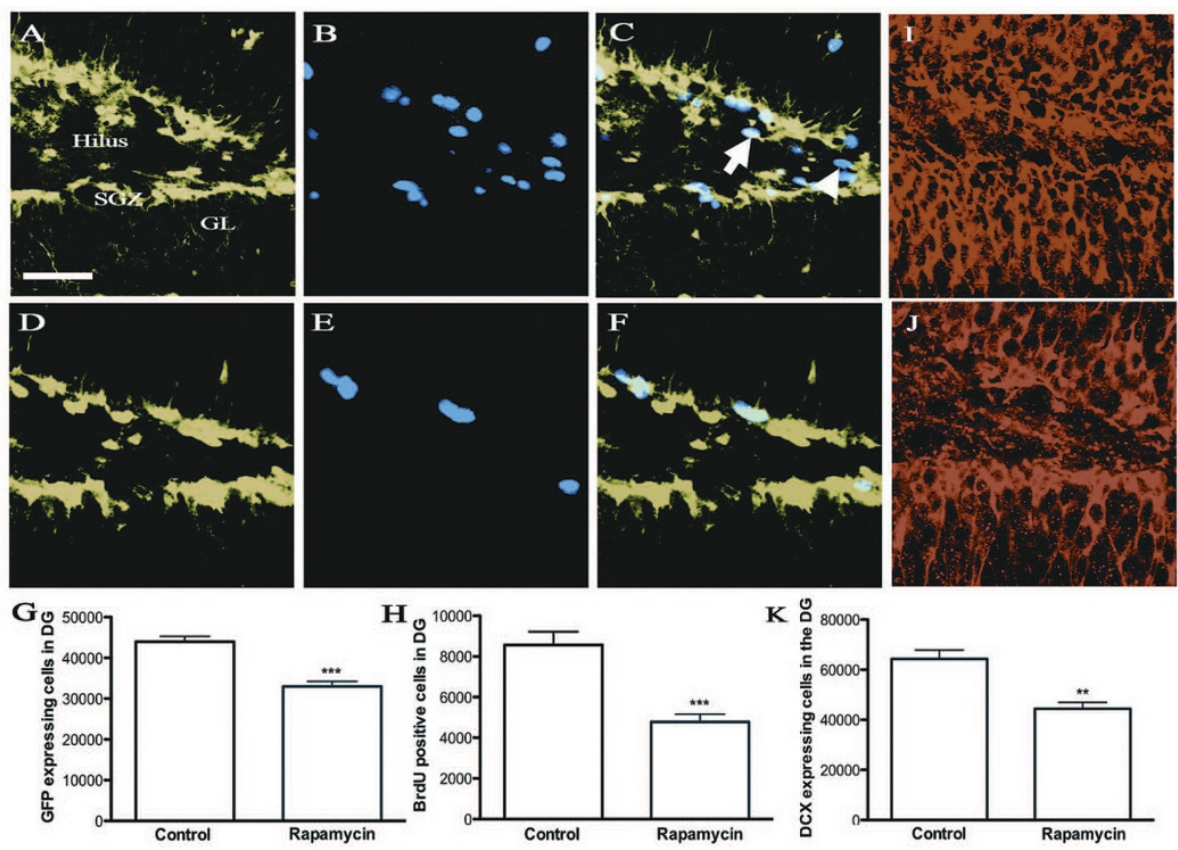

Figure 5. Changes in the progenitor cell population within the DG at P14 after rapamycin injection. Representative pictures of GFP, BrdU, and DCX expression in DG from mice under control $(A, B, I)$ and rapamycin-treated conditions $(D, E, J)$, respectively, showing significantly decreased numbers of GFP-positive $(G)$, BrdU-positive cells $(H)$ and DCX-positive cells $(K)$ with rapamycin treatment as quantified by design-based stereology. The merged picture for control $(C)$ and rapamycin treated $(F)$ shows BrdU-positive cells that are colocalized (arrow) and ones that are not (arrowhead). $p$ values for GFP, BrdU, and DCX are significant. ${ }^{* *} p<$ 0.001. Error bars represent SD, and scale bars represent $35 \mu \mathrm{M}$. early and late progenitors and actively dividing cells compared with the control animals. These differences were similar to the changes observed in $\mathrm{CH}$ (Fig. $5 A-K$ ). The decrease in GFP-expressing early progenitors, as quantified by the number of GFP-positive cells was $25 \%(43,965 \pm 1,365$ in control versus $33,026 \pm 1,196$ in rapamycin treated), whereas in DCX-expressing late progenitors it was 30\% (64,316 $\pm 7,801$ in control versus 44,415 $\pm 5,072$ in rapamycin treated), whereas in BrdU-expressing actively dividing cells it was reduced by $45 \%$ ( $8554 \pm 667$ in control versus 4,764 \pm 369 in rapamycin treated). The percentage decrease is greater than that seen in animals exposed to $\mathrm{CH}$ at a similar time point.

\section{DISCUSSION}

$\mathrm{CH}$ is an important risk factor for infants with cyanotic congenital heart disease, and brain injury is a major cause of their neurological morbidity in these infants (21). The animal model of $\mathrm{CH}$ used in this study closely mimics the degree of hypoxia seen in infants with cyanotic heart disease (2) and has been widely used to study the effects of $\mathrm{CH}$ on the developing brain such as alteration in NMDA receptor expression and function and alterations in energy metabolism (2,22). Despite the clear association between $\mathrm{CH}$ and brain dysfunction, mechanisms underlying these observations remain unclear. 
Here, we demonstrate a significant decrease in neurogenesis in the DG in mice exposed to $\mathrm{CH}$. The DG is one of the two most well-characterized areas where neurogenesis occurs postnatally (4). The peak of DG neurogenesis occurs in mice, a time when the developing brain may be particularly sensitive to insults such as hypoxia (23). It is well known that the microenvironment affects the proliferative potential of a variety of organ-specific stem/progenitor cells (24). This may explain the $\sim 50 \%$ reduction in the progenitor stem cell population in the chronic hypoxic group at P7 when the microenvironment of the neurogenic niche is altered at the peak of its proliferative potential. The differentiation potential of the progenitor population suggests that at P7, most of the progenitors differentiate into neurons (20). Therefore, our observations demonstrating significant decreases in the neuronal pool of the developing $\mathrm{DG}$ under $\mathrm{CH}$ during this time are not unexpected. This is further confirmed by the decrease in the DCX-expressing late progenitor cell population and a decrease in the volume of the neuronal layer at P14 in $\mathrm{CH}$. Altering the microenvironment during this phase of rapid development causes long-term impairment in the proliferative potential as evidenced by a decrease in the number of actively dividing cells at P56. This decrease seen at P56 suggest long-lasting impairment of proliferative potential or may represent just a lag in proliferation from the catch up growth seen when animals are removed from $\mathrm{CH}$. The long-term effect of this change remains unknown.

To gain insight into one of the potential mechanisms underlying our observations, we examined the status of the mTOR signaling pathway. In the nervous system, mTOR has been implicated in axonal and dendritic development and pruning of dendritic arborizations $(14,15)$, which we have previously shown to be altered during exposure to $\mathrm{CH}$ (3). In cultured hippocampal neurons, 4E-BP1 is present in the dendrites and at synaptic sites (25). The mTOR pathway is a key regulator of cell growth and proliferation and has been implicated in neurogenesis and differentiation of progenitor stem cells (26). In a model of global ischemia, neurogenesis is induced through CD95 and its receptor CD95L (27), a process known to be mediated through activation of the mTOR pathway.

Because hypoxia is a major regulator of the mTOR pathway, we quantified levels of total and phosphorylated S6 and $4 \mathrm{E}-\mathrm{BP} 1$ as readouts of mTOR activity, and demonstrated that phosphorylated levels of both are decreased in the cortex in $\mathrm{CH}$. The significant decrease in the phosphorylation levels of both substrates in the cortex suggests that inhibition of mTOR activity may be an adaptive mechanism to limit energy expenditure. This is supported by a decrease in creatine levels and altered phosphocreatine to creatine ratio seen by proton spectroscopy in our model (2).

Finally, using rapamycin as a specific inhibitor of the mTOR pathway, we demonstrated a significant decrease in the early and late progenitors as well as the actively dividing cells seen in CH. Rapamycin has been shown to decrease neurogenesis and subsequent mossy fiber sprouting in an adult mouse model of kainic acid-induced seizures (28). This model of seizure activity has been shown to be regulated by mTOR activation. Moreover, rapamycin has been used to decrease mTOR-induced neuronal differentiation of progenitor stem cells induced by insulin (26). Thus, it seems that the overall decrease in the progenitor stem pool in $\mathrm{CH}$ is mediated at least in part through mTOR signaling.

In conclusion, we have demonstrated that $\mathrm{CH}$ causes a significant decrease in the progenitor stem pool and that this decrease may in part be mediated through mTOR signaling. Progenitor stem cells in the developing brain continuously add to the pool of functional neurons and other cell types throughout life (29). Such integration into the functional pool is likely critical for proper cognitive function, particularly early in hippocampal development. It has been shown that following other forms of injury such as traumatic brain injury and hypoxia-ischemia, there is an increase in the progenitor pool that helps replace the loss of functional neurons $(10,11)$. However, in $\mathrm{CH}$, the long-term decrease in this progenitor pool may put the developing brain at greater risk for learning disorders later in life.

Acknowledgments. We thank Gui Zhang for technical assistance, Dr. Raghu Rao for critical review, and Dr. Renee McKay for manuscript preparation.

\section{REFERENCES}

1. Avishai-Eliner S, Brunson KL, Sandman CA, Baram TZ 2002 Stressed-out, or in (utero)? Trends Neurosci 25:518-524

2. Raman L, Tkac I, Ennis K, Georgieff MK, Gruetter R, Rao R 2005 In vivo effect of chronic hypoxia on the neurochemical profile of the developing rat hippocampus. Brain Res Dev Brain Res 156:202-209

3. Raman L, Hamilton KL, Gewirtz JC, Rao R 2008 Effects of chronic hypoxia in developing rats on dendritic morphology of the CA1 subarea of the hippocampus and on fear-potentiated startle. Brain Res 1190:167-174

4. Altman J, Das GD 1965 Autoradiographic and histological evidence of postnatal hippocampal neurogenesis in rats. J Comp Neurol 124:319-335

5. Kempermann G, Gage FH 2000 Neurogenesis in the adult hippocampus. Novartis Found Symp 231:220-235

6. Frederiksen K, McKay RD 1988 Proliferation and differentiation of rat neuroepithelial precursor cells in vivo. J Neurosci 8:1144-1151

7. Fukuda S, Kato F, Tozuka Y, Yamaguchi M, Miyamoto Y, Hisatsune T 2003 Two distinct subpopulations of nestin-positive cells in adult mouse dentate gyrus. J Neurosci 23:9357-9366

8. Kunze A, Grass S, Witte OW, Yamaguchi M, Kempermann G, Redecker C 2006 Proliferative response of distinct hippocampal progenitor cell populations after cortical infarcts in the adult brain. Neurobiol Dis 21:324-332

9. Brown J, Cooper-Kuhn CM, Kempermann G, Van Praag H, Winkler J, Gage FH, Kuhn HG 2003 Enriched environment and physical activity stimulate hippocampal but not olfactory bulb neurogenesis. Eur J Neurosci 17:2042-2046

10. Miles DK, Kernie SG 2008 Hypoxic-ischemic brain injury activates early hippocampal stem/progenitor cells to replace vulnerable neuroblasts. Hippocampus 18:793806

11. Kernie SG, Erwin TM, Parada LF 2001 Brain remodeling due to neuronal and astrocytic proliferation after controlled cortical injury in mice. J Neurosci Res 66:317-326

12. Liu L, Cash TP, Jones RG, Keith B, Thompson CB, Simon MC 2006 Hypoxiainduced energy stress regulates mRNA translation and cell growth. Mol Cell 21:521-531

13. Swiech L, Perycz M, Malik A, Jaworski J 2008 Role of mTOR in physiology and pathology of the nervous system. Biochim Biophys Acta 1784:116-132

14. Jaworski J, Spangler S, Seeburg DP, Hoogenraad CC, Sheng M 2005 Control of dendritic arborization by the phosphoinositide-3'-kinase-Akt-mammalian target of rapamycin pathway. J Neurosci 25:11300-11312

15. Kumar V, Zhang MX, Swank MW, Kunz J, Wu GY 2005 Regulation of dendritic morphogenesis by Ras-PI3K-Akt-mTOR and Ras-MAPK signaling pathways. J Neurosci 25:11288-11299

16. Pei JJ, Hugon J 2008 mTOR-dependent signalling in Alzheimer's disease. J Cell Mol Med 12:2525-2532

17. Sarkar S, Rubinsztein DC 2008 Huntington's disease: degradation of mutant huntingtin by autophagy. FEBS J 275:4263-4270

18. Klann E 2009 Thwarting dyskinesia by targeting mTORC1. Sci Signal 2:pe42

19. Yu TS, Dandekar M, Monteggia LM, Parada LF, Kernie SG 2005 Temporally regulated expression of Cre recombinase in neural stem cells. Genesis 41:147-153 
20. Gilley JA, Yang CP, Kernie SG 2011 Developmental profiling of postnatal dentate gyrus progenitors provides evidence for dynamic cell-autonomous regulation. Hippocampus $21: 33-47$

21. Bass JL, Corwin M, Gozal D, Moore C, Nishida H, Parker S, Schonwald A, Wilker RE, Stehle S, Kinane TB 2004 The effect of chronic or intermittent hypoxia on cognition in childhood: a review of the evidence. Pediatrics 114:805816

22. Pichiule P, Chavez JC, Boero J, Arregui A 1996 Chronic hypoxia induces modification of the N-methyl-D-aspartate receptor in rat brain. Neurosci Lett 218:83-86

23. Schlessinger AR, Cowan WM, Gottlieb DI 1975 An autoradiographic study of the time of origin and the pattern of granule cell migration in the dentate gyrus of the rat. J Comp Neurol 159:149-175

24. Ivasenko IN, Klestova OV, Arkad'eva GE, Almazov VA 1990 [Role of stroma microenvironment in the regulation of bone marrow hemopoiesis after curantyl administration.] Biull Eksp Biol Med 110:98-100
25. Tang SJ, Reis G, Kang H, Gingras AC, Sonenberg N, Schuman EM 2002 A rapamycin-sensitive signaling pathway contributes to long-term synaptic plasticity in the hippocampus. Proc Natl Acad Sci U S A 99:467-472

26. Han J, Wang B, Xiao Z, Gao Y, Zhao Y, Zhang J, Chen B, Wang X, Dai J 2008 Mammalian target of rapamycin (mTOR) is involved in the neuronal differentiation of neural progenitors induced by insulin. Mol Cell Neurosci 39:118-124

27. Corsini NS, Sancho-Martinez I, Laudenklos S, Glagow D, Kumar S, Letellier E, Koch P, Teodorczyk M, Kleber S, Klussmann S, Wiestler B, Brustle O, Mueller W, Gieffers C, Hill O, Thiemann M, Seedorf M, Gretz N, Sprengel R, Celikel T, Martin-Villalba A 2009 The death receptor CD95 activates adult neural stem cells for working memory formation and brain repair. Cell Stem Cell 5:178-190

28. Zeng LH, Rensing NR, Wong M 2009 The mammalian target of rapamycin signaling pathway mediates epileptogenesis in a model of temporal lobe epilepsy. J Neurosci 29:6964-6972

29. Li G, Pleasure SJ 2010 Ongoing interplay between the neural network and neurogenesis in the adult hippocampus. Curr Opin Neurobiol 20:126-133 